\title{
Apreciación jurídica de la salud en la perspectiva de los derechos humanos
}

\section{Legal assessment of health from the perspective of human rights}

\author{
Jorge Luis Restrepo Pimienta ${ }^{1}$
}

\section{RESUMEN}

El presente artículo pretende realizar un análisis del derecho a la salud a partir de su categoría de fundamental y desde el enfoque holístico de los derechos humanos, para lo cual se ha precisado acudir a referentes contemporáneos en el ámbito de la teoría jurídica de los derechos humanos. Asimismo, se plantean conceptos normativos y jurisprudenciales entorno al derecho a la salud desde la perspectiva internacional.

\section{Palabras clave:}

Derecho a la salud, Derechos humanos, seguridad social en salud, salud pública.

\section{ABSTRACT}

The present article intends to carry out an analysis of the right to health from its category of fundamental and from the holistic approach of human rights, for which it has been necessary to go to contemporary references in the field of legal theory of human rights . Likewise, normative and jurisprudential concepts are raised regarding the right to health from the international perspective.

\section{Key words:}

Right to health, Human rights, social security in health, public health.

\footnotetext{
${ }^{1}$ Abogado, PhD en Derecho, McS Derecho Profundización Seguridad Social, Esp Derecho Laboral y Seguridad Social, Par Académico COLCIENCIAS, CNA y CONACES, Docente de Carrera Universidad del Atlántico, Investigador Asociado. Correo: jorgerestrepo@mail.uniatlantico.edu.co ISSN: 2346-2787

Recibido: 3 de Abril de 2018

Aprobado: 28 de Mayo de 2018.
} 


\section{Introducción.}

Pretender hablar de salud en cuanto a su mirada jurídica es empezar a decir que está soportado en el marco de las fuentes del derecho y obligaciones establecidos en los entes de derecho internacional público que regulan la materia y en los nacionales que legislan al respecto, cimentados en marcos culturales, morales y políticos.

Así las cosas se puede decir que la salud en su calidad de condición humana individual está regulada en leyes, códigos, presupuestos económicos y políticos, regímenes, sistemas, reglas técnicas, principios y valores, esto se afirma dado a que la categoría de derecho humano fundamental que tiene la salud y definición de principios y valores históricos instructores de la salud pública que aseguran su pleno goce obligan a considerar sus unidades, que aunque se desagregan por una razón formativa, hacer parte de una unidad inherente (Escudero, 2011, pág. 80).

Cabría destacar dentro del mundo de la historia antigua, la salud en cuanto a su reconocimiento y relevancia por filósofos griegos, a partir de que es un bien inherente a la naturaleza y condición humana, específicamente para Aristóteles citado por Escudero (2011), "si los hombres como seres humanos poseen derechos que les son propios, entonces tiene derecho de gozar de buena salud", con esta valoración se inicia el extenso universos de ideas, preceptos, pautas, normas y cánones acerca del derecho humano fundamental a la salud.

En cuanto a la descripción histórica de acontecimientos sociales jurídicos y políticos en cuanto a la garantía y derecho a la salud en basamentos según Paredes Hernàndez (2003), únicamente se tenía como bien jurídico protegido por el derecho penal y civil, no se concebía como derecho autónomo; de este modo evolucionista en las dos últimas décadas del siglo XIX, aparecieron las leyes de 
protección en Europa, primeros principios de los derechos sociales, y primera expresión del sistema de seguridad social en el mundo; consecutivamente con el aparecimiento del Estado benefactor, la salud era una prestación social a cargo del ente estatal, por tanto se tenía la salud concebida como servicio público.

De esta manera cabe empezar a definir la naturaleza del derecho a la salud, el cual es posible ubicarlo como uno de los Derechos fundamentales y como consecuencia de los Derechos Humanos, los primeros son derechos de carácter netamente constitucional, es decir, del orden publico interno y en este sentido es importante comprender que estos derechos poseen unas garantías suplementarias al interior de la nación, por lo tanto regulan entre los ciudadanos determinadas relaciones a fin de que estas puedan ser eficaces de manera directa e inmediata pues todas las personas miembros de una nación están obligadas o sometidas al cumplimiento de estos derechos y deberán responder en la medida que los violenten, ya que es a partir de este orden de valores que se proyecta una vida social óptima.

De manera consecutiva y Jerárquica existe la segunda categoría de derechos aquí planteada es entendida como un cumulo de derechos que cuentan con un fundamento ético-político y del derecho internacional, a fin de entender esto separadamente, resulta acertado determinar que son éticos en cuanto regulan la convivencia pacífica de los asociados a la nación, tienen un alcance político en la medida que buscan la detracción de los poderes políticos y por último y lo cual les da una amplia significación son del derecho internacional público, en este sentido son instrumentos de los Estados, pues a través de esta categoría de derechos se crean obligaciones tanto positivas como negativas. 
En este marco dialógico de esbozo y debido a que al ser reconocidos por determinadas naciones se convierten en normas de rigor dentro del ordenamiento jurídico, ya que así tengan su origen o se hubieren positivizado la norma por medio de diferentes organismos internacionales adquieren en países como Colombia un rango constitucional, por tal razón las autoridades deben velar por el respeto y la garantía de estos tan importantes derechos, pues son los Estados los responsables por la vulneración de alguno de los tratados internacionales a los que se hubieren obligado.

Seguidamente con el objeto de referenciar y esclarecer tasaciones jurídico políticas con respecto a los derechos humanos se parte de la concepción de praxis social, estos representan el emblema de un Estado determinado con un modelo garantista con denominación antropocéntrica, denominado Social de Derechos, el cual debe propender por el bienestar de sus asociados, puesto que se institucionalizo en países europeos y americanos, con miras a brindar a sus habitantes las mayores y mejores condiciones justas y dignas de vida en todos los campos y aspectos del ser; ya que de acuerdo con la evolución que ha sufrido los derechos a la salud, sanidad y la seguridad social en salud, la protección al mismo se ha visto afectada y es así como han comenzado a surgir una normatividad en busca de la defensa de los trabajadores (Barona, 2014).

En la actualidad los Derechos Humanos juegan un papel fundamental en todo tipo de Estado, ya que este tipo de derechos se han estructurado como uno de los pilares fundamentales de las sociedades modernas a nivel global; pero estos no solo son los únicos existentes sino que los Estados modernos han contextualizado más precisamente una serie de garantías constitucionales a sus ciudadanos a través de los derechos fundamentales estipulados en la Constitución Política. 
Ahora bien los Derechos fundamentales que vendrán a ser el pilar principal del Estado social de Derecho acompañado de la Dignidad humana, son la razón principal para que se fundamente la existencia del Estado Social de Derechos y no solo el Estado de Derechos. (Chinchilla, 1999). Es importante resaltar que existe una diversidad de autores y teorías, incluso hasta de Estados que consideran que los derechos humanos se equiparan a los derechos fundamentales, aunque la teoría constitucional Occidental se ha esforzado por puntualizar la divergencia de sus significados; varios tratadista les han dado la misma significación, equiparado e incluyendo constitucionalmente los derechos que se reconocen en la Carta Magna como fundamentales a los mismos derechos humanos (Chinchilla, 1999).

Existe una pluralidad de autores que definen los derechos humanos y los derechos fundamentales como el mismo concepto jurídico de derechos en forma material y formal, en tales consideraciones se encuentran autores como el Jurista Italiano Ferrajoli (2011, pág. 35), que menciona una definición general de derechos humanos:

Todos aquellos derechos subjetivos que corresponden universalmente a todos los seres humanos dotados del estatus de persona, de ciudadanos o personas con capacidad de obrar, entendiendo por derecho subjetivo cualquier expectativa positiva (de prestaciones) o negativa (de no sufrir lesiones) adscritas a un sujeto por una norma jurídica."

En concordancia y tratando de ilustrar de la mejor manera con el acápite anterior, se observan otras definiciones en el mismo sentido el ilustre profesor Boliviano Rivera Santivañez (2007, pág. 121), que también funde ambos conceptos al afirmar que: 
... Como parte del proceso de Judicialización de los derechos humanos el amparo constitucional debe constituirse en la vía tutelar efectiva e idónea para otorgar una protección inmediata a las personas, o en su caso, a los grupos sociales, cuyos derechos humanos son vulnerados de manera ilegal e indebida. Ello implica que el amparo constitucional otorgue tutela a todos los derechos humanos consagrados en las constituciones de los Estados así como en los tratados, convenciones o pactos internacionales sobre la materia."

Dentro del continente americano se encuentra en Chile el Tratadista Cea quien también considera que la distinción de derechos humanos y derechos fundamentales no configura diferencia alguna por ello manifiesta la Caracterización de los derechos fundamentales en lo que refiere a los derechos humanos en la constitución (Cea Egaña, 2004, pág. 31).

En el continente Europeo con detención en la republica de España el jurista Diez Picazo (2000, pág. 227) También considera que no existe dicotomía alguna en el significado de los derechos fundamentales y los derechos humanos, al expresar que:

... Ciertamente el concreto régimen jurídico y en especial, los mecanismos de protección de los derechos variaran según sea la norma en la cual estén reconocidos; pero ello no autoriza a olvidar que se trata siempre de derechos tendentes a salvaguardar unos mismos valores que, desde un punto de vista moral y político, se consideran básicos para la convivencia humana."

Ahora bien, contrario sensu al concepto que tienen los autores que se mencionaron en párrafos anteriores, existen otros autores que abordan la temática 
de manera diferente, considerando que los derechos fundamentales y los derechos humanos no tienen definiciones convergentes, sino que por el contrario tienen dos significados diferentes, entre los cuales encontramos tratadistas tales como el tratadista Duràn Ribera (1995. , pág. 37) que sobre la temática en cuestión expresa:

“... Es posible sostener que bajo la expresión derechos fundamentales se designa a los derechos garantizados por la Constitución y que en cambio, la denominación derechos humanos, hace referencia a derechos garantizados por normas internacionales. Las primeras tienen como fuente de producción al legislador constituyente, y las segundas, a los Estados y organismos internacionales."

En concordancia con el párrafo anterior también se encuentra a la ilustre profesora Española Garrido (2007), que explica la dicotomía de los significados de derechos humanos y derechos fundamentales de la siguiente manera:

El termino derechos Humanos es el más tradicional en el uso referido a los derechos positivados que, en el plano internacional, se traducen en exigencias morales o naturales reclamadas como derechos básicos e, incluso, es el vocablo que se usa para hacer alusión a las exigencias que, con determinadas condiciones y relacionadas con la dignidad, la libertad y la igualdad de la persona, aún no han alcanzado un estatuto jurídico-positivo. ... En cambio, a los derechos humanos los esgrimimos a una pretensión moral fuerte que debe atenderse para hacer posible una vida digna ... la voz derechos humanos es vaga en alto grado, lo que representa una nota de la función histórica que pretende erigirse en criterio que mida la legitimidad de un modelo político y, consiguiente, que justifique la obediencia a sus normas ... Mas cuando hablamos de derechos fundamentales, la expresión es más precisa, siendo los derechos que se recogen en la constitución de los Estados y son 
apoyados por el ordenamiento jurídico y el sistema de garantías correspondientes. (Garrido Gòmez, 2007, pág. 15)"

En continuación con lo referido con anterioridad, también se puede apreciar el concepto del tratadista (Pèrez Luño, 1991, pág. 22) que considera que se debe "reservar la denominación 'derechos fundamentales' para designar los derechos humanos positivados a nivel interno, en tanto que la fórmula' derechos humanos' es la más usual en el plano de las declaraciones y convenciones internacionales".

Dentro de este orden de ideas es oportuno señalar las apreciaciones acerca de sintetizar, confusiones, diferencias, similitudes, analogías, divergencias que se aproximen a la construcción o edificación de un conjunto de declaraciones que enriquezcan las dimensiones epistémicas, cognitivas, ilustrativas y pragmáticas a la vez que dichos preceptos jurídicos, lo cuales se constituyen en raíz y centro de las garantías de los ciudadanos, en el espacio y medio de los diversos sistemas sociales, es por ello que en los término Pérez (1998., pág. 10) Expresa:

Derechos humanos» $\mathrm{y}$ "derechos fundamentales» son utilizados, muchas veces, como sinónimos. Sin embargo, no han faltado tentativas doctrinales encaminadas a explicar el respectivo alcance de ambas expresiones. Así, se ha hecho hincapié en la propensión doctrinal y normativa a reservar el término "derechos fundamentales» para designar los derechos positivados a nivel interno, en tanto que la fórmula "derechos humanos" sería la más usual para denominar los derechos naturales positivados en las declaraciones y convenciones internacionales, así como a aquellas exigencias básicas relacionadas con la dignidad, libertad e igualdad de la persona que no han alcanzado un estatuto jurídico positivo. 


\section{Referentes teóricos derecho humano a la salud}

Continuando con un marco científico constitucional, político y social, entre dichos lineamientos cabe resaltar que para Bockenforde (1994, pág. 46), se destacan las teorías sobre la interpretación, compresión, aplicación, proyección de los derechos fundamentales, teniendo como partida, base fundamental y cimiento al desarrollo esquematizado analítico del modelo de Estado, de la siguiente manera:

Las principales teorías de los derechos fundamentales desde las que se determina hoy, de forma alternativa o combinadas, la interpretación de los derechos fundamentales, son las siguientes: la teoría liberal o del Estado de derecho burgués de los derechos fundamentales, la teoría institucional de los derechos fundamentales, la teoría axiológica de los derechos fundamentales, la teoría democrático-funcional de los derechos fundamentales, y la teoría del Estado social" pág 46.

Dentro de esta perspectiva que viene abordando de funcionalismo estructural y la delimitación con referente a las instituciones jurídicas dentro de un sistema social, abarca así destacar la teoría institucional funcionalista de los derechos fundamentales, la cual es tratada por Niklas Luhmann, el cual de manera detallada y concreta esboza según Pérez (1991, pág. 11) la siguiente postura:

Tales derechos fundamentales no deben ser considerados ni como facultades emanadas de la naturaleza humana (iusnaturalismo), ni como límites a la actuación del poder público (liberalismo), sino como instituciones, esto es, subsistemas encaminados a cumplir unas determinadas funciones que permiten: de un lado, la diferenciación de los roles sociales, y, de otro, garantizan el desarrollo de la actividad estatal 
Ahora bien, en virtud de la Constitución Política de 1991 desde un ámbito institucional funcionalista, para demostrar en la práctica Colombiana, precisamente de la lectura del artículo 93, que reza de la siguiente manera: "Los tratados y convenios internacionales ratificados por el Congreso, que reconocen los derechos humanos y que prohíben su limitación en los estados de excepción, prevalecen en el orden interno. Los derechos y deberes consagrados en esta Carta, se interpretarán de conformidad con los tratados internacionales sobre derechos humanos ratificados por Colombia.", esto en el sistema de salud significa la relevancia y respecto a los entes de derecho internacional público que hacen posible las tendencias y defensas de los derechos humanos en la operatividad de los mismos sin tener en cuenta el modelo de Estado teniendo en cuanta la ideología política y económica, lo que interesa en puesto funcional que estos ejercen en la Institución (Peña Peña, 2007.)

Se puede rematar que dentro del funcionalismo estructural en la teoría institucional, que entonces no se puede hablar o departir acerca de los derechos humanos y derechos fundamentales en estricto sentido o formalmente hablando; todo esto en cuanto existen derechos humanos que está prohibida su limitación y por tal razón no se pueden incluir al bloque de constitucionalidad para obtener el rango de constitucional que tienen los derechos fundamentales en su totalidad.

Con el análisis precedente frente a la funcionalidad y estructuralidad que representa los derecho fundamentales, nos lleva a pensar que en el Estado Colombiano en materia de los derechos fundamentales se conciben como aquellos derechos inherentes a la personalidad esto quiere decir que, bajo ninguna situación pueden ser limitados en el territorio nacional; por tales razones se puede concluir que en términos y entendimiento de la Constitución Política, los derechos humanos y los derechos humanos no configuran un mismo significado 
entendido desde una perspectiva formal, aunque guarden una estrecha relación como derechos de las personas (Peña Peña, 2007, pag 43).

En este mismo sentido, para Uprimy (1996, pág. 26), considera que "los derechos humanos, los derechos constitucionales y los derechos fundamentales no son, en estricto sentido, conceptos sinónimos", el tratadista considera que el contenido legal de este tipo de derechos que puede ser muy similar, pero el significado de cada uno de estos tipos de derechos tiene consecuencias normativas diferentes".

Con base en lo antes expuesto se puede decir que Rodrigo Uprimny explica en esta línea que los derechos humanos son un concepto ético-político del derecho internacional, mientras que los derechos fundamentales son una categoría del derecho constitucional, esto es, del derecho público interno.

Se fortalece esta compresión teórica por medio de (Alexy, 1995, pág. 65), señala sobre la temática que los derechos humanos son supra positivos o morales, y que al ser incorporados en una Constitución "los derechos humanos se positivizan como derechos fundamentales", en tales consideraciones se aprecia que no hay para Colombia una conceptualización idéntica entre los significados de los derechos humanos y los derechos fundamentales en términos formales, ya que materialmente puede que si guarden la idéntica significación.

Por demás se puede decir que formalmente configuran derechos diferentes y en muchos casos comparten derechos iguales, los efectos que producen son diferentes, todo esto de acuerdo al ámbito que se usen; pues si hablamos en el ámbito internacional el termino correcto será el de derechos humanos y en el ámbito interno será el de los derechos fundamentales. No obstante es necesario 
aclarar que no todos los derechos humanos a nivel interno son fundamentales y mucho menos tienen supremacía constitucional, por tal razón se evidencia su diferencia formal desde los párrafos de la Constitución Política de Colombia cuando los discrimina frente a los estados de excepción (Cantor Rey, 2006).

Por ende, cabe destacar entonces que en lo relacionado a la salud es válido aducir dentro de la dogmática jurídica, que en las naciones unidas se exprese "Es mi aspiración que la salud sea vista no como una bendición deseable, sino como un derecho humano por el cual luchar" (Kofi Annan, Secretario General de las Naciones Unidas. "It is my aspiration that health will finally be seen not as a blessing to be wished for, but as a human right to be fought for").

Asimismo se puede decir que la realidad en torno a los derechos humanos, no se cumple en un derecho específico, como el de la salud, si no que rige una noción de defensa y respeto al espíritu que fundamenta el derecho internacional de los derechos humanos. Evidentemente éste se encuentra incorporado al ordenamiento constitucional, jurídico, ético, bioético, deontológico, institucional y por tanto obliga al Estado como parte de estos instrumentos internacionales, pero no se podría considerar que la sociedad colombiana haya asumido los derechos humanos como una visión rectora ni de las relaciones del Estado con las personas, ni del valor de la dignidad humana individual (Gòmez Gallego, 2008).

De tal forma, que la realización del derecho a la salud probablemente adolece de las mismas debilidades que cualquier otro derecho en el país y su realización se enfrenta con los mismos obstáculos. Podría decirse que las debilidades que afectan su potencial como derecho derivan de la "salud" de los derechos humanos (Gòmez Gallego, 2008). 
La anterior consideración, que da cuenta de las debilidades presentes en el entorno en términos de conciencia y compromiso desde una lógica de derechos humanos subyacente a las políticas públicas, sociales y reflejada en el lento desarrollo normativo de cada una de las obligaciones internacionales adquiridas, afirma la importancia de que jurídicamente el derecho esté protegido de manera sólida, y sustenta la necesidad de mecanismos estrictos de vigilancia y control como elementos necesarios para su efectividad; tal como se conceptualiza en el derecho internacional de los derechos humanos, el derecho a la salud es un bien jurídico exigible al Estado como parte de la consagración de los Derechos Económicos, Sociales y Culturales "DESC" (Yamin, 2006).

Partiendo de supuestos normativos vigentes y conceptos precedentes cabe resaltar que El Estado colombiano quedó obligado a partir de la ratificación de los instrumentos de derecho internacional, bajo el principio de Pacta Sunt Servanda, lo que en el caso de la salud, como todos los derechos humanos, deriva de la noción de respeto a la dignidad humana y significa un avance en los compromisos morales y políticos de las sociedades (Cantor Rey, 2006).

Para continuar connotando apreciaciones ilustrativas y estableciendo bases cognoscitivas racionadas se destaca que los Derechos Económicos, Sociales y Culturales, se rigen por los principios que definen todos los derechos consagrados en el derecho internacional, como son la universalidad, indivisibilidad e interdependencia (Chinchilla, 1999).

Con la base de lo antes expuesto se puede decir que tales principios hacen que se apliquen las mismas exigencias para su realización que aquellas que se imponen en el respeto a los derechos civiles y políticos, es decir, medidas adecuadas y efectivas de promoción, respeto y garantía, con la misma obligación de resultado. 
Esto se entiende en términos de resultado "progresivo", a diferencia de los derechos civiles y políticos para los que se exige una efectividad inmediata (Chinchilla, 1999).

Es así que la naturaleza jurídica del Derecho a la Salud, se inicia partiendo de la protección especial Estado de normalidad y condiciones óptimas del ser humano, es así que en el derecho internacional de los derechos humanos tiende a generar debate en dos (2) sentidos relacionados con su naturaleza jurídica; por una parte es un derecho fundamental en sí mismo, o si lo es por conexidad con el derecho a la vida y otros derechos; por otra parte, la salud como derecho se inscribe dentro de los DESCA (Derechos económicos, sociales, culturales y ambientales), lo cual implica unos términos de amparo y realización de los derechos comunes a este género, con algunas diferencias frente a los que obligan a los Estados en relación con los derechos civiles y políticos, lo que se concluye con ello es que es un objetivo social ineludible del goce universal, que conlleva a la obligación de participación activa de todos los miembros de la comunidad y el Estado (Escudero, 2011).

Entonces en aras de precisar la salud en perspectiva de derechos humanos se enmarca en la normatividad internacional y en consecuencia en el cumplimiento de las obligaciones que son responsabilidad de carácter social, jurídico y político de los Estados sin distinción de la elección de lineamientos y modelos, solo por el hecho $o$ acto de adquirirlas al momento de suscribir los pactos internacionales pertinentes al tema, es por ello que cabría decir en este orden de aseveraciones que este goza de una protección y reconocimiento normativo, facultativo y político en escala mundial el universal, esto indica que este derecho es una construcción social dialéctica en donde la población participa (Escudero, 2011). 
Tanto los derechos reconocidos expresa e inicialmente como fundamentales, es decir aquellos que asumen la protección del individuo frente al mismo poder del Estado, bien sea en la defensa de su libertad, vida, igualdad ante la ley, etc., como los derechos prestacionales, la salud entre ellos, que requieren para su efectiva realización la intervención activa y positiva del Estado, de lo cual se destaca que es una construcción ordenada multicasualidad de factores sociales, científicos, ecónomos y tecnológicos (Escudero, 2011).

El desarrollo de la legislación e institucionalidad adecuadas o la asignación consecuente de recursos, hacen parte de la exigencia del derecho para que su realización no sea un simple enunciado sujeto a las posibilidades acordes al grado de desarrollo de los distintos Estados y las decisiones de poder, o a la voluntad política de las diferentes administraciones, entonces por esta razón y circunstancia de carácter político expresa (Gòmez Gallego, 2008, pág. 216):

"El derecho a la salud es el más inacabado de los derechos, en el sentido de que sus posibilidades dependen de una técnica que no termina de crecer y perfeccionarse, y por ende el abanico de exigibilidad. No concretar este núcleo por su complejidad es dejar indefinida la exigencia concreta del Estado sobre ese mínimo de servicios irrenunciables"

Algunos sectores doctrinarios y políticos tienden a cuestionar la fuerza jurídica de los derechos sociales entre ellos la salud y su entendimiento como derechos exigibles, con el argumento de que se trata de derechos de realización progresiva, que requieren la asignación de recursos y desarrollos legales, de manera que su satisfacción depende de la voluntad de los órganos políticos de decisión; para otro sector de la doctrina y para el Comité de Derechos Económicos, Sociales y Culturales "Comité DESC", esto no es así, pues se ha mostrado que hay obligaciones de realización inmediata en relación con los derechos sociales y que para el caso de salud son también determinadas (Gòmez Gallego, 2008). 
Como lo han señalado numerosos autores, esa diferencia debe relativizarse, pues los DESC también tienen obligaciones de inmediato cumplimiento (como la prohibición de discriminación), y el mandato de progresividad no es una autorización para la inacción de los Estados (Abramovich, Víctor y Courtis, Christian 2002, P. 24-37).

Conceptos doctrinales, normativo jurídicos y jurisprudenciales entorno al derecho a la salud desde la perspectiva internacional.

En cuanto a la compresión jurídica normativa del derecho a la salud se retomando las primeras fuentes que sean las de carácter internacional para luego terminar con las estatales de carácter legal y convencional, entonces una vez precisada la naturaleza del derecho a la salud, procederemos a recordar brevemente los principales tratados que reconocen este derecho. $Y$ es que existe una serie de instrumentos jurídicos internacionales en los cuales está expresamente reconocido el derecho a la salud; ellos constituyen el parámetro que delimita el contenido del derecho, la responsabilidad del Estado y el ámbito de protección que puede exigir toda persona; seguidamente expresadas en el cuadro:

Tabla 13.

Políticas Internacionales de salud.

Norma

La Declaración Universal de Derechos

\section{Concepto Jurídico Internacional}

Protege explícitamente el derecho a la salud al decir que: "Toda persona tiene 


\section{Norma}

Humanos

El Pacto Internacional de Derechos Civiles y Políticos

El Pacto Internacional de Derechos Económicos Sociales y Culturales
Observación General No. 14 relativa al Artículo 12
Concepto Jurídico Internacional

derecho a un nivel de vida adecuado que le asegure, así como a su familia, la salud y en especial la alimentación, el vestido, la vivienda, la asistencia médica y los servicios sociales necesarios".

El Artículo 7, dispone que "nadie será sometido a torturas ni a penas o tratos crueles, inhumanos o degradantes. En particular, nadie será sometido sin su libre consentimiento a experimentos médicos científicos". Este pacto fue aprobado por Colombia mediante la Ley 74 de 1968

Art 12 Los Estados Partes en el presente Pacto reconocen el derecho de toda persona al disfrute del más alto nivel posible de salud física y mental.

Que define la naturaleza, el contenido y alcance del derecho a partir de la inclusión de elementos y procedimientos complementarios, inherentes a la afirmación de la salud como "derecho humano fundamental e indispensable para el ejercicio de los demás derechos humanos".

Habla del compromiso de adoptar medidas pertinentes, no limitadas ni condicionadas por otras consideraciones, por ejemplo, de recursos.

Reconoce el derecho a la salud pública, la asistencia médica, la seguridad

La Convención Internacional sobre la Eliminación de Todas las Formas de 


\section{Norma}

Discriminación Racial (Ley 22 de 1981)

La Convención sobre la Eliminación de Todas las Formas de Discriminación contra la Mujer, (Ley 1 de 1981)
Concepto Jurídico Internacional

social y los servicios sociales.

Incluye el derecho a la protección de la salud y a la seguridad en las condiciones de trabajo, incluso la salvaguardia de la función de reproducción; el acceso a los servicios que se refieren a la planificación familiar, el período posterior al parto, y la nutrición adecuada durante el embarazo y lactancia.

La Convención sobre los Derechos del Niño (Ley 12 de 1991)

La Declaración Americana de los

Derechos y Deberes del Hombre consagra en el artículo XI

La Convención Americana de Derechos Humanos
Artículo 24 la plena aplicación del derecho a la salud y la adopción de medidas mediante, entre otras cosas, la aplicación de la tecnología disponible, el suministro de alimentos nutritivos adecuados y agua potable salubre; la atención sanitaria prenatal y posnatal apropiada a las madres; y el desarrollo de atención sanitaria preventiva.

Requiere la adopción de las medidas eficaces y apropiadas posibles para abolir las prácticas tradicionales que sean perjudiciales para la salud del niño.

El derecho de toda persona a la preservación de la salud y el bienestar

Aunque no hace alusión expresa al derecho, sí afirma en su artículo 26 que los Estados partes se comprometen "a adoptar providencias, tanto a nivel interno como mediante la cooperación internacional, especialmente económica y técnica" para lograr progresivamente la plena efectividad de los derechos 


\section{Norma}

Protocolo Adicional a la Convención Americana sobre Derechos Humanos en materia de Derechos Económicos, Sociales y Culturales, o Protocolo de San Salvador suscrito en 1988 , aprobado por Colombia en 1996 y que entró en vigor en 1999
Concepto Jurídico Internacional

contenidos en la Carta de la

Organización de los Estados

Americanos, que en su artículo 33 hace

referencia al derecho a la salud

(Arbeláez, 2009, Pág. 53)

Artículo 10:

1. Toda persona tiene derecho a la salud, entendida como el disfrute del más alto nivel de bienestar físico, mental y social

Fuente: Diseño del Autor

Luego de la reseña analítica de normas jurídicas a nivel internacional que a la vez han sido ratificas por muchos Estados entre ellos Colombia, cabe ilustrar para precisar diferencias y similitudes, desafíos y retos, arreglos y contradicciones, programas y acciones, entre otros es el cuadro esquemático planteado por la OMS, el cual permite asimilar en cuanto al conocimiento, saber y realidad social y proyectista acerca del derecho a la salud, derecho sanitario y derecho a la seguridad social en salud así: 


\section{"EI derecho a la salud"}

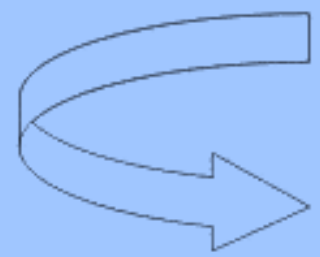

Factores determinantes

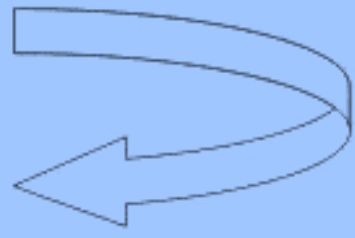

Atención de salud

agua, saneamiento, alimentos, nutrición, vivienda, condiciones sanas en el trabajo y el medio ambiente, educación, información, etc.

Disponibilidad, accesibilidad, aceptabilidad, calidad

(Observación general N 14 del Comité de Dereches Económices, Sociales y Culturales 


\section{Referencias.}

Alexy, R. (1995). Teoría del discurso y Derechos Humanos. Bogotá:: (Trad Luis Villar Borda)Universidad Externado de Colombia.

Bockenforde, E. W. (1994). Escritos Sobre Derechos Fundamentales. Valencia : Baden Nomos.

Cantor Rey, E. (2006). El bloque de constitucionalidad aplicación de tratados internacionales de derechos humanos. Estudios Constitucionales, 288-334.

Cea Egaña, J. L. (2004). Derecho Constitucional Chileno. Derechos, Deberes y Garantías. Santiago de Chile: Universidad Católica de Chile.

Chinchilla, H. T. (1999). Queson y cuales son los derechos Fundamentales. Bogota: Temis.

Diez Picazo, L. M. (2000). Aproximaciones a la idea de los Derechos Fundamentales. Revista Peruana de Derecho Constitucional, 225-226.

Duràn Ribera, W. R. (1995. ). Curso de Derechos Fundamentales. Madrid. : Universidad Carlos Tercero de Madrid - Boletín oficial del Estado, .

Escudero, C. H. (2011). Principios Orientadores de la Salud Pùblica desde el Derecho a la Salud. En G. Malagòn Londoño, Salud Pùblica Perspectivas (págs. 79-113). Bogotà : Panamericana .

Ferrajoli, L. (2011). Constitucionalismo principialista. Roma : Universidad de Roma III.

Garrido Gòmez, M. I. (2007). Derechos Fundamentales y Estado Social y Democrático de Derecho. Madrid : Dieles .

Gòmez Gallego, R. (2008). La Dignidad Humana en el proceso salud-enfermedad. Bogotà: Univerisdad del Rosario . 
Paredes Hernàndez, N. (2003). El Derecho a la Salud: Su situación en Colombia. Bogotá: Antropo.

Pérez Luño, A. .. (1998.). Los Derechos Fundamentales. Madrid: Tecnos.

Pèrez Luño, A. E. (1991). Derechos humanos, estado de derecho y constitución. Madrid : Tecnos.

Rivera Santivañez, J. A. (2007). Temas de Derecho Procesal Constitucional. Cochabamba: Kipus.

Uprimny Yepes, R. ". (1996). Algunas reflexiones sobre la responsabilidad por la violación de los derechos humanos en la Constitución". Bogotà: Univerisdad Nacional de Colombia.

Yamin, A. E. (2006). Los Derechos Sociales, econòmicos y Culturales en amèrica Latina. Mexico . 